\title{
New Formulas for Facilitating Osculatory Interpolation
}

\author{
Herbert E. Salzer
}

\begin{abstract}
Hermite's $n$-point osculatory interpolation formula for equally spaced arguments at intervals of $h$, employing the function and its derivative is very much more accurate than the corresponding $n$-point Lagrangian formula and considerably more accurate than even the $2 n$-point Lagrangian formula at intervals of $h$. Also it is specially suited for interpolation in many functions (e. g., Bessel, probability) that are tabulated with their derivative. To avoid the tremendous amount of labor in calculating the coefficients of $f_{i}$ and $f_{i}^{\prime}$ in the forms that they are usually given, Hermite's formula is expressed as
\end{abstract}

$$
f\left(x_{0}+p h\right)=\sum_{i}\left(\alpha_{i} f_{i}+\beta_{i} h f_{i}^{\prime}\right) / \sum_{i} \alpha_{i}+R_{2 n}(p),
$$

where

and where

$$
\alpha_{i} \equiv a_{i} /(p-i)^{2}+b_{i} /(p-i), \beta_{i} \equiv a_{i} /(p-i),
$$

being

$$
a_{i}=k(n) /\left\{\underset{j=-[(n-1) / 2]}{\left.\Pi^{\prime}\right]}(i-j)\right\}^{2}, b_{i}=-2 L_{i}^{(n)^{\prime}}(i) a_{i}, L_{i}^{(n)}(p)
$$

$$
\underset{j=-[(n-1) / 2]}{\left.\Pi^{\prime}\right]}(p-j) / \underset{j=-[(n-1) / 2]}{\Pi^{\prime}}(i-j) .
$$

The constant $k(n)$, which may be picked arbitrarily, is here chosen to make $a_{i}$ and $b_{i}$ integers. The exact values of $a_{i}$ and $b_{i}$ are given for $n=2(1) 11, i=-[(n-1) / 2]$ to $[n / 2]$ so that this formula can be applied exactly for any polynomial up to the 21 st degree. A schedule gives approximate upper bounds for the coefficients of $f^{(2 n)}(\xi) h^{2 n} \sim \Delta^{2 n} f(x)$ in $R(p)$.

When a function $f(x)$ and its first derivative are known at $n$ points $x_{i}, i=1,2, \ldots, n$, a highly accurate interpolation formula due to Hermite is given by

$$
f(x)=\sum_{i=1}^{n}\left\{L_{i}^{(n)}(x)\right\}^{2}\left\{1-2 L_{i}^{(n)}\left(x_{i}\right)\left(x-x_{i}\right)\right\} f\left(x_{i}\right)+\sum_{i=1}^{n}\left\{L_{i}^{(n)}(x)\right\}^{2}\left(x-x_{i}\right) f^{\prime}\left(x_{i}\right)+R_{2 n}(x),
$$

where

$$
L_{i}^{(n)}(x)=\prod_{j=1}^{n}\left(x-x_{j}\right) / \Pi_{j=1}^{n}\left(x_{i}-x_{j}\right), \quad j=i \text { is absent from } \Pi^{\prime},
$$

and

$$
R_{2 n}(x)=f^{(2 n)}(\xi)\left\{\prod_{j=1}^{n}\left(x-x_{\jmath}\right)\right\}^{2} /(2 n) !, \quad \text { least } x_{i} \leq \xi \leq \text { greatest } x_{i} .
$$

Thus (1) is exact whenever $f(x)$ is a polynomial of degree less than or equal to $2 n-1$.

When the points $x_{i}$ are equally spaced at intervals of $h$, it is customary to alter the notation in $x_{i}$, letting $i$ run from $-[(n-1) / 2]$ to $[n / 2]$ instead of 1 to $n$, where $[m]$ denotes the largest integer not exceeding $m$. Then it is convenient to choose a variable $p$ given by $x=x_{0}+p h$ and to let $x_{i}=x_{0}+i h$. Also, $f(x)=f\left(x_{0}+p h\right) \equiv f p \equiv f, f\left(x_{i}\right) \equiv f_{i}$, and $f^{\prime}\left(x_{i}\right) \equiv f_{i}^{\prime}$. Then (1), for $f(x)$ considered as a function of $p$, is expressible as

$f\left(x_{0}+p h\right)=\sum_{i=-[(n-1) / 2]}^{[n / 2]}\left\{L_{i}^{(n)}(p)\right\}^{2}\left\{1-2 L_{i}^{(n)^{\prime}}(i)(p-i)\right\} f_{i}+\sum_{i=-[(n-1) / 2]}^{\lfloor n / 2]}\left\{L_{i}^{(n)}(p)\right\}^{2}(p-i) h f_{i}^{\prime}+R_{2 n}(p)$,

where now

$$
L_{i}^{(n)}(p)=\underset{j=-[(n-1) / 2]}{\stackrel{[n / 2]}{\Pi^{\prime}}}(p-j) / \underset{j=-[(n-1) / 2]}{\stackrel{[n / 2]}{\Pi^{\prime}}}(i-j),
$$

and

$$
R_{2 n}(p)=f^{(2 n)}(\xi) h^{2 n}\{\underset{j=-I(n-1) / 2]}{[n / 2]}(p-j)\}^{2} /(2 n) !, \quad x_{-[(n-1) / 2]} \leq \xi \leq x_{[n / 2]} .
$$


There are many advantages in the use of (1) or (4) over the ordinary Lagrangian interpolation formula given (for equal spacing) by

$$
f\left(x_{0}+p h\right)=\sum_{i=-[(n-1) / 2]}^{[n / 2]} L_{i}^{(n)}(p) f_{i}+R_{n}(p),
$$

where

$$
R_{n}(p)=f^{(n)}(\xi) h^{n} \prod_{j=-[(n-1) / 2]}^{[n / 2]}(p-j) / n !, \quad x_{-[(n-1) / 2]} \leq \xi \leq x_{[n / 2]} .
$$

Thus letting $\prod_{j=-[(n-1) / 2]}^{[n / 2]}(p-j)$ be denoted by $L^{(n)}(p)$, and recalling that for reasonably small $h$ we have approximately

$$
f^{(m)}(\xi) h^{m} \sim \Delta^{m} f
$$

where $\Delta^{m} f$ is the approximate $m$ th difference of the tabulated $f(x)$, the remainder term for (7) is of the order of $\Delta^{n} f L^{(n)}(p) / n$ !, whereas that for (4) is of the order of $\Delta^{2 n} f\left\{L^{(n)}(p)\right\}^{2} /(2 n)$ !. Apart from the fact that $\Delta^{2 n} f$ is usually very much smaller than $\Delta^{n} f$, the factor $\left\{L^{(n)}(p)\right\}^{2} /(2 n)$ ! is equal to the square of $L^{(n)}(p) / n$ ! (where the latter is usually very small and less than unity so that its square is ever so much smaller) multiplied by the very small quantity $n ! /(n+1)$ ... $(2 n)$. The user can appreciate the improvement by comparing the approximate upper bounds for the multiplier of $f^{(2 n)}(\xi) h^{2 n}$ in $R_{2 n}(p)$ of (4) which are tabulated in the schedule at the end, with the approximate upper bounds for the multiplier of $f^{(n)}(\xi) h^{n}$ in $R_{n}(p)$ of $(7)$, which are tabulated in [3, p. xvi $]^{1}$ and from which this present schedule was calculated. Thus it will be apparent that (4) is a very much more accurate formula than (7). Of course, we are comparing (4), a confluent form of a $2 n$-point formula, with (7), which is only an $n$-point formula.

But it is important to note than even if (4) is compared with formula (7) taken for $2 n$ points at intervals of $h$, instead of $n$ points, the remainder term would differ from that in (4) (apart from a different $\xi$ in $f^{(2 n)}(\xi)$ ) by the presence of the factor $L^{(2 n)}(p)$ instead of the factor $\left\{L^{(n)}(p)\right\}^{2}$, which has a very much smaller upper bound than the $L^{(2 n)}(p)$, showing that wherever it is possible to be used, the $n$-point Hermite osculating interpolation formula is much to be preferred, regarding accuracy, to a $2 n$-point Lagrangian interpolation formula at the same interval $h$. 'This last statement becomes intuitively plausible when the osculating interpolation formula for $n$ points at intervals of $h$ is regarded as a confluent form of a $2 n$-point Lagrangian formula whose $2 n$ points lie within a range of $n h$ so that the "average interval" between those $2 n$ points is only half the interval of $h$ for the usual $2 n$-point Lagrangian formula. Thus the upper bound for the remainder term of the $n$-point osculating formula would be expected to be of the order of $\left(1 / 2^{2 n}\right)$ th of the upper bound for the remainder term of the $2 n$-point Lagrangian formula; actual estimates show it to be even considerably smaller. For example, the 2-, 3-, 4-, and 5-point osculating formulas have error terms whose upper bounds are around $1 / 16$ th, $1 / 110,1 / 640$, and $1 / 3000$ of the respective upper bounds of the error terms in the 4-, 6-, 8-, and 10-point Lagrangian formulas.

A second important advantage in (4) is that it is suited for use with very many tables where the derivative of the function is tabulated alongside of the function itself. For example, it is useful in tables of Bessel functions of the first and second kind $[4,5,6]$ which give $J_{1}(x)=$ $-J_{0}^{\prime}(x), Y_{1}(x)=-Y_{0}^{\prime}(x)$, and probability functions [7], and in numerous tables of more elementary functions and their integrals, such as tables of sine, cosine, or exponential integrals $[8,9]$, where the derivative is very easy to obtain.

However, the use of (1) or (4) in the form usually presented [1, 2] requires a considerable amount of computational labor which mounts considerably as the number of points $x_{i}$ increases. It is the purpose of this present article to provide a means of using (4) with a small fraction of the labor involved in the direct calculation of the coefficients of $f_{i}$ and $f_{i}^{\prime}$. The idea

\footnotetext{
1 Figures in brackets indicate the literature references at the end of this paper.
} 
behind this method goes back to a scheme first used by W. J. Taylor for calculating Lagrangian interpolation coefficients for functions tabulated at real equidistant arguments [10], and which was generalized by the present writer for functions tabulated at nonequidistant arguments [11], and also for complex arguments whether in Cartesian [12] or polar form [13, 14], and finally even for functions that are interpolable by expressions that are not transformable into polynomials [15]. Recently, the writer in looking for some way to reduce the amount of work in using (4), observed that Taylor's idea could be extended also to the calculation of osculating interpolation coefficients. In place of extensive tables of the $(2 n-1)$ th degree polynomial coefficients of $f_{i}$ and $f_{i}^{\prime}$ in (4), one requires for each separate $n$ only some fixed quantities $a_{i}$ and $b_{i}$, which are exact integers and are tabulated below. To see this, one merely expresses (4) as

$$
f\left(x_{0}+p h\right)=\left\{L^{(n)}(p)\right\}^{2} \sum_{i=-\lceil(n-1) / 2]}^{[n / 2 \mid}\left\{\left(\frac{A_{i}^{2}}{(p-i)^{2}}-\frac{2 L_{i}^{(n)}(i) A_{i}^{2}}{(p-i)}\right) f_{i}+\frac{A_{i}^{2}}{(p-i)} h f_{i}^{\prime}\right\}+R_{2 n}(p),
$$

where $^{2}$

$$
A_{i} \equiv 1 / \underset{j=-[(n-1) / 2]}{\stackrel{[n / 2]}{\Pi^{\prime}}}(i-j)
$$

Now the right member of (4) or (10) without the $R_{2 n}(p)$ gives the expression for a $(2 n-1)$ th degree polynomial, which, with its derivative, assumes preassigned values of $f_{i}$ and $f_{i}^{\prime}$ at $x=x_{i}$, and moreover that polynomial is uniquely determined by the $f_{i}$ and $f_{i}^{\prime}$. For proof of uniqueness see [1, p. 85-86], where T. Fort gives a demonstration of the unique existence of a more general osculating formula. His proof is practically complete save for the explicit indication that the mode of representation of any $(m n-1)$ th degree polynomial which is given at the bottom of page 85 is always possible (which is fairly obvious). Now we make use of this uniqueness of representation by putting $f(x) \equiv 1$ into $(10)$, so that both $f_{i}^{\prime}$ and $R_{2 n}(p)$ are zero, $f_{i}=1$, and we get

Thus from (10) and (12),

$$
\left\{L^{n}(p)\right\}^{2}==\sum_{i=-[(n-1) / 2]}^{[n / 2]}\left(\frac{A_{i}^{2}}{(p-i)^{2}}-\frac{2 L_{i}^{(n) \prime}(i) A_{i}^{2}}{p-i}\right) .
$$

$$
f\left(x_{0}+p h\right)=\frac{\sum_{i=-[(n-1) / 2]}^{[n / 2]}\left\{\left(\frac{a_{i}}{(p-i)^{2}}+\frac{b_{i}}{(p-i)}\right) f_{i}+\frac{a_{i} h}{(p-i)} f_{i}^{\prime}\right\}}{\sum_{i=-[(n-1) / 2]}^{[n / 2]}\left(\frac{a_{i}}{(p-i)^{2}}+\frac{b_{i}}{(p-i)}\right)}+R_{2 n}(p),
$$

where $a_{i}$ and $b_{i}$ are given by

$$
\begin{aligned}
& a_{i}=k(n) A_{i}^{2}, \\
& b_{i}=k(n)\left\{-2 L_{i}^{(n)^{\prime}}(i) A_{i}^{2}\right\},
\end{aligned}
$$

and where $k(n)$ is any suitably chosen constant of proportionality that depends only upon $n$ : In the present case the $k(n)$ was chosen as to give exact integral values for $a_{i}$ and $b_{i}$ instead of rational fractional values.

It is simplest to think of the approximation to $f(x)$ in the concise form

$$
f \sim \frac{\Sigma\left(\alpha_{i} f_{i}+\beta_{i} h f_{i}{ }^{\prime}\right)}{\Sigma \alpha_{i}},
$$

where

and

$$
\alpha_{i} \equiv a_{i} /(p-i)^{2}+b_{i} /(p-i)
$$

$$
\beta_{i} \equiv a_{i} /(p-i)
$$

${ }_{2}^{2}$ The dependence upon $n$ of $A_{i}$, as well as of $a_{i}$ and $b_{i}$ given below, is not indicated, so as to avoid cumbersome notation. 
In using (16), (17), and (18) with a desk calculator, it is easiest to first divide $a_{i}$ by $p-i$ to get $\beta_{i}$, which is next both multiplied by $h f_{i}{ }^{\prime}$ and increased by $b_{i}$. The latter, or $\beta_{i}+b_{i}$, is again divided by $(p-i)$ to give $\alpha_{i}$, from which one obtains both $\alpha_{i} f_{i}$ and $\Sigma \alpha_{i}$ and finally $\Sigma\left(\alpha_{i} f_{i}+\beta_{i} h f_{i}{ }^{\prime}\right) / \Sigma \alpha_{i}$.

The computation of the quantities $a_{i}$ and $b_{i}$ was quite straightforward. Since $A_{i}=$ $(-1)^{[n / 2]-i}\left(\begin{array}{c}n-1 \\ i+[(n-1) / 2]\end{array}\right) /(n-1)$ !, instead of $A_{i}{ }^{2}$, the proportional quantities $\left(\begin{array}{c}n-1 \\ i+[(n-1) / 2]\end{array}\right)^{2}$ were calculated. Then they were multiplied by the $-2 L_{i}{ }^{(n) \prime}(i)$, which were calculated by differentiating the explicit polynomial expressions $L_{i}{ }^{(n)}(p)$ and then setting $p=i$. All fractions in $-2\left(\begin{array}{c}n-1 \\ i+[(n-1) / 2]\end{array}\right)^{2} L_{i}^{(n) \prime}(i)$ were cleared by multiplication of these quantities, as well as the $\left(\begin{array}{c}n-1 \\ i+[(n-1) / 2]\end{array}\right)^{2}$, by some suitable integer, for each $n$, to yield the exact integral values for $a_{i}$ and $b_{i}$, which are tabulated below. The $a_{i}$ and $b_{i}$ were checked by both recomputation and by use in an example for every $n$ where the answer was known exactly and where the computation by (13) (or the equivalent (16), (17), and (18)) doing the work in decimal form to avoid too much labor, gave agreement to 10 significant figures.

The schedule giving the approximate upper bounds for the coefficients of $f^{(2 n)}(\xi) h^{2 n} \sim \Delta^{2 n} f$ in the error term $R_{2 n}(p)$, (see (4) with (6), or (13)), namely, the quantities $\left\{L^{(n)}(p)\right\}^{2} /(2 n)$ !, was calculated from the approximate upper bounds for $L^{(n)}(p) / n$ ! given in a schedule in [3, p. xvi], by squaring the entries in the latter and multiplying by $n ! /(n+1) \ldots(2 n)$. Thus, since the $L^{(n)}(p) / n$ ! was tabulated only approximately, in some cases to only one significant figure, some of the upper bounds given here for $\left\{L^{(n)}(p)\right\}^{2} /(2 n)$ ! are not at all precise. For example, if we take a rounded 0.01 and square it to obtain 0.0001 , the true value of that square may be only one-fourth as large or over twice as large, depending upon whether the 0.01 was rounded from 0.0051 or 0.0149 . Hence in the schedule below, in some cases for the larger number of points, only the order of magnitude of an upper bound for the $\left\{L^{(n)}(p)\right\}^{2} /(2 n)$ ! is indicated. But more precise determination will hardly be needed there due to the extreme accuracy that is surely indicated even within the range of the uncertainty.

[1] T. Fort, Finite differences, p. 85-88 (Clarendon Press, Oxford, England, 1948).

[2] J. F. Steffensen, Interpolation, p. 33-34 (Williams \& Wilkins, Baltimore, Md., 1927).

[3] National Bureau of Standards, Tables of Lagrangian interpolation coefficients (Columbia University Press, New York, N. Y., 1944; 2d ed. 1948).

[4] National Bureau of Standards, Table of the Bessel functions $J_{0}(z)$ and $J_{1}(z)$ for complex arguments (Columbia University Press, New York, N. Y., 1943; 2d ed. 1947).

[5] National Bureau of Standards, Table of the Bessel functions $Y_{0}(z)$ and $Y_{1}(z)$ for complex arguments (Columbia University Press, New York, N. Y., 1950).

[6] Harvard Computation Laboratory, The annals of the computation laboratory of Harvard University III, Tables of the Bessel functions of the first kind of orders zero and one (Harvard University Press, Cambridge, Mass., 1947).

[7] Tables of probability functions, NBS MT8, I (1941); NBS MT14, II (1942), 2d ed. (1948).

[8] Tables of sine, cosine, and exponential integrals, NBS MT5; NBS MT6 (1940).

[9] Table of sine and cosine integrals for arguments from 10 to 100, NBS MT12 (1942).

[10] W. J. Taylor, Method of Lagrangian curvilinear interpolation, J. Research NBS 35, 151-155 (1945) RP1667.

[11] H. E. Salzer, Checking and interpolation of functions tabulated at certain irregular logarithmic intervals, J. Research NBS 46, 74-77 (1951) RP2177.

[12] H. E. Salzer, Formulas for complex Cartesian interpolation of higher degree, J. Math. Phys. 28, 200-203 (1949).

[13] H. E. Salzer, Alternative formulas for direct interpolation of a complex function tabulated along equidistant circular arcs, J. Math. Phys. 26, 56-61 (1947).

[14] H. E. Salzer, Coefficients for polar complex interpolation, J. Math. Phys. 29, 96-104 (1950).

[15] H. E. Salzer, Coefficients for facilitating trigonometric interpolation, J. Math. Phys. 27, 274-278 (1949). 
Table of $a_{i}$ and $b_{i}$

\begin{tabular}{|c|c|c|c|c|c|c|c|c|c|c|c|c|}
\hline \multicolumn{4}{|c|}{ Two-point } & \multicolumn{4}{|c|}{ Seven-point } & \multicolumn{5}{|c|}{ Ten-point } \\
\hline$a_{0}$ & 1 & $b_{0}$ & 2 & $a_{-3}$ & 10 & $b_{-3}$ & 49 & $a_{-4}$ & & 1260 & $b_{-4}$ & 7129 \\
\hline$a_{1}$ & 1 & $b_{1}$ & -2 & $a_{-2}$ & 360 & $b_{-2}$ & 924 & $a_{-3}$ & 1 & 02060 & $b_{-3}$ & 350649 \\
\hline \multirow{2}{*}{\multicolumn{4}{|c|}{ Three-point }} & $a_{-1}$ & 2250 & $b_{-1}$ & 2625 & $a_{-2}$ & 16 & 32960 & $b_{-2}$ & 3569184 \\
\hline & & & & $a_{0}$ & 4000 & $b_{0}$ & 0 & $a_{-1}$ & 88 & 90560 & $b_{-1}$ & 10965024 \\
\hline$a_{-1}$ & 1 & $b_{-1}$ & 3 & $a_{1}$ & 2250 & $b_{1}$ & -2625 & $a_{0}$ & 200 & 03760 & $b_{0}$ & $\begin{array}{lll}80 & 01504\end{array}$ \\
\hline$a_{0}$ & 4 & $b_{0}$ & 0 & $a_{2}$ & 360 & $b_{2}$ & -924 & $a_{1}$ & 200 & 03760 & $b_{1}$ & -8001504 \\
\hline$a_{1}$ & 1 & $b_{1}$ & -3 & $a_{3}$ & 10 & $b_{3}$ & -49 & $a_{2}$ & 88 & 90560 & $b_{2}$ & -10965024 \\
\hline \multicolumn{4}{|c|}{ Four-point } & \multicolumn{4}{|c|}{ Eight-point } & $\begin{array}{l}a_{3} \\
a_{4}\end{array}$ & $\begin{array}{r}16 \\
1\end{array}$ & $\begin{array}{l}32960 \\
02060\end{array}$ & $\begin{array}{l}b_{3} \\
b_{4}\end{array}$ & $\begin{array}{r}-3569184 \\
-350649\end{array}$ \\
\hline$a_{-1}$ & 3 & $b_{-1}$ & 11 & $a_{-3}$ & 70 & $b_{-3}$ & 363 & $a_{5}$ & & 1260 & $b_{5}$ & -7129 \\
\hline$a_{0}$ & 27 & $b_{0}$ & 27 & $a_{-2}$ & 3430 & $b_{-2}$ & 9947 & \multirow{2}{*}{\multicolumn{5}{|c|}{ Eleven-point }} \\
\hline$a_{1}$ & 27 & $b_{1}$ & -27 & $a_{-1}$ & 30870 & $b_{-1}$ & 48363 & & & & & \\
\hline$a_{2}$ & 3 & $b_{2}$ & -11 & $a_{0}$ & 85750 & $t_{0}$ & 42875 & $a_{-5}$ & & 1260 & $b_{-5}$ & 7381 \\
\hline \multirow{2}{*}{\multicolumn{4}{|c|}{ Five-point }} & $a_{1}$ & 85750 & $b_{1}$ & -42875 & $a_{-4}$ & 1 & 26000 & $b_{-4}$ & 460900 \\
\hline & & & & $a_{2}$ & 30870 & $b_{2}$ & -48363 & $a_{-3}$ & 25 & 51500 & $b_{-3}$ & $62 \quad 14725$ \\
\hline$a_{-2}$ & 6 & $b_{-2}$ & 25 & $a_{3}$ & 3430 & $b_{3}$ & -9947 & $a_{-2}$ & 181 & 44000 & $b_{-2}$ & 27561600 \\
\hline$a_{-1}$ & 96 & $b_{-1}$ & 160 & $a_{4}$ & 70 & $b_{4}$ & -363 & $a_{-1}$ & 555 & 66000 & $b_{-1}$ & 40748400 \\
\hline$a_{0}$ & 216 & $b_{0}$ & 0 & \multirow{2}{*}{\multicolumn{4}{|c|}{ Nine-point }} & $a_{0}$ & 800 & 15040 & $b_{0}$ & 0 \\
\hline$a_{1}$ & 96 & $b_{1}$ & -160 & & & & & $a_{1}$ & 555 & 66000 & $b_{1}$ & -40748400 \\
\hline$a_{2}$ & 6 & $b_{2}$ & -25 & $a_{-4}$ & 140 & $b_{-4}$ & 761 & $a_{2}$ & 181 & 44000 & $b_{2}$ & -27561600 \\
\hline \multirow{2}{*}{\multicolumn{4}{|c|}{ Six-point }} & $a_{-3}$ & 8960 & $b_{-3}$ & 28544 & $a_{3}$ & 25 & 51500 & $b_{3}$ & $-62 \quad 14725$ \\
\hline & & & & $a_{-2}$ & 109760 & $b_{-2}$ & 208544 & $a_{4}$ & & 26000 & $b_{4}$ & -460900 \\
\hline$a_{-2}$ & 30 & $b_{-2}$ & 137 & $a_{-1}$ & 439040 & $b_{-1}$ & 395136 & $a_{5}$ & & 1260 & $b_{5}$ & -7381 \\
\hline$a_{-1}$ & 750 & $b_{-1}$ & 1625 & $a_{0}$ & 686000 & $b_{0}$ & 0 & & & & & \\
\hline$a_{0}$ & 3000 & $b_{0}$ & 2000 & $a_{1}$ & 439040 & $b_{1}$ & -395136 & & & & & \\
\hline$a_{1}$ & 3000 & $b_{1}$ & -2000 & $a_{2}$ & 109760 & $b_{2}$ & -208544 & & & & & \\
\hline$a_{2}$ & 750 & $b_{2}$ & -1625 & $a_{3}$ & 8960 & $b_{3}$ & -28544 & & & & & \\
\hline$a_{3}$ & 30 & $b_{3}$ & -137 & $a_{4}$ & 140 & $b_{4}$ & -761 & & & & & \\
\hline
\end{tabular}


Schedule of approximate upper bounds for $\left\{L^{(n)}(p)\right\}^{2} /(2 n)$ !

(Figures in parentheses indicate the number of zeros between the decimal point and the first significant digit.)

\begin{tabular}{|c|c|c|c|c|c|}
\hline Range of $p_{-}$ & $\{0<p<1$ & $\begin{array}{r}-1<p<0 \\
1<p<2\end{array}$ & $\begin{array}{r}-2<p<-1 \\
2<p<3\end{array}$ & $\begin{array}{r}-3<p<-2 \\
3<p<4\end{array}$ & $\begin{array}{r}-4<p<-3 \\
4<p<5\end{array}$ \\
\hline $\begin{array}{l}\text { Two-point } \\
\text { Four-point } \\
\text { Six-point } \\
\text { Eight-point } \\
\text { Ten-point }\end{array}$ & $\begin{array}{l}0026 \\
.(5) 82 \\
\cdot(7) 26 \\
.(10) 94 \\
.(12) 34\end{array}$ & $\begin{array}{l}.(4) 25 \\
.(7) 55 \\
.(9) 15 \\
.(12) 49\end{array}$ & $\begin{array}{l}\text { ( }(6) 62 \\
.(9) 85 \\
.(11) 18\end{array}$ & $\begin{array}{l}\cdot(7) 20 \\
\cdot(10) 18\end{array}$ & . (9) 78 \\
\hline Range of $p_{\ldots} \ldots \ldots$ & $0<|p|<1$ & $1<|p|<2$ & $2<|p|<3$ & $3<|p|<4$ & $4<|p|<5$ \\
\hline $\begin{array}{l}\text { Three-point } \\
\text { Five-point } \\
\text { Seven-point } \\
\text { Nine-point } \\
\text { Eleven-point }\end{array}$ & $\begin{array}{l}00021 \\
\cdot(6) 57 \\
\cdot(8) 18 \\
\cdot(11) 60 \\
.(13) 6\end{array}$ & $\begin{array}{l}.(5) 38 \\
\cdot(8) 62 \\
\cdot(10) 15 \\
.(13) 6\end{array}$ & $\begin{array}{r}\text { (6) } 11 \\
.(9) 12 \\
.(12) 2\end{array}$ & $\begin{array}{l}\text { ( } 8) 40 \\
\text { (11) } 6\end{array}$ & . (9) 1 \\
\hline
\end{tabular}

Washington, May 12, 1953. 